\title{
Enhanced Assessment Technology and Neurocognitive Aspects of Specific Learning Disorder with Impairment in Mathematics.
}

\author{
https://doi.org/10.3991/ijep.v8i1.7370 \\ Marios A. Pappas $\left.{ }^{(}\right)$, Eugenia Malli, Vasiliki Kalpidi, Athanasios S. Drigas \\ National Center for Scientific Research "Demokritos", Agia Paraskevi, Attica, Greece \\ mpapeit.demokritos.gr
}

\begin{abstract}
Specific Learning Disorder with impairment in Mathematics (Developmental Dyscalculia) is a complex learning disorder which affects arithmetic skills, symbolic magnitude processing, alertness, flexibility in problem solving and maintained attention. Neuro-cognitive studies revealed that such difficulties in children with DD could be related to poor Working Memory and attention deficits. Furthermore, neuroimaging studies indicate that brain structure differences in children with DD compared to typically developing children could affect mathematical performance. In this study we present the cognitive profile of Dyscalculia, as well as the neuropsychological aspects of the deficit, with special reference to the utilization of enhanced assessment technology such as computerized neuropsychological tools and neuroimaging techniques.
\end{abstract}

Keywords-Specific Learning Disorders, Mathematics, Developmental Dyscalculia, Working Memory, Cognition, Attention.

\section{Introduction}

Numbers are everywhere in our lives. From starting our day with the question "What time is it?", to making calculations at the market. All these functions are related to number form area of brain and this area exists in both hemispheres [1]. Both parts of brain (left and right) are neural correlates of number processing. Researchers have proved that there are brain regions connected to magnitude processing and Developmental Dyscalculia [2].

The term dyscalculia was introduced in the 1940s, but it didn't become fully recognized until the 1974 when a Czechoslovakian researcher Ladislav Kosc introduced it as Developmental Dyscalculia [3]. According to DSM-V children with Specific Learning Disorder with impairment in Mathematics, face difficulties related to number understanding, recalling numerical facts, performing numerical computations and mathematical reasoning. This is a neuro-developmental disorder with biological origins which occurs during the first school years and is not due to mental disorders [4]. 
We define two different types of dyscalculia, the one is developmental dyscalculia in which someone was born with it. The other is the commonly held type as acquired dyscalculia, a disorder that arise later in somebody's life, usually as a result of an injury with brain damage. The prevalence of developmental dyscalculia was estimated 1 to $6 \%$ in a population of 1004 children aged 7-10 years in United Kingdom [5]. In addition, equal numbers of boys and girls were diagnosed with developmental dyscalculia, so there is not a discrimination by gender in the particular disorder.

Rapin et al [6] distinguished the following four stages of arithmetic skills: 1.In infancy, babies come into first contact with colours, shapes and quantities. 2. In preschool ages, toddlers start counting, without having consciousness of numbers and their uniqueness. 3. In first grade of school, children learn basic operations, like addition, subtraction with one or two-digit numbers and mental calculation. 4. In elementary school, teenagers are able to handle numbers more effectively, do basic operations and learn algorithmic procedures for solving problems. Included in the above publication, the results of 378 eight years old-intelligence over 85-children in German school were presented. $6 \%$ of the participants were diagnosed with dyscalculia, but only $1.8 \%$ were diagnosed with pure dyscalculia and the rest $4.2 \%$ had dyscalculia in combination with another developmental disorder (dyslexia, ADHD, etc).

At the front side of brain (prefrontal lobe), circuits contribute to decision making and non-automated behaviors. During the stages of learning and maturation, activities are transferred from the front area to the parietal region. One of the most famous methods of neuroimaging is fMRI (functional Magnetic Resonance Imaging). The above method refers to an imaging brain area, which is activated during the execution of certain functions. There is a minimum of five functional networks in the human brain: attention, language, memory, recognition, working memory networks [7].

At the left and right hemishpere, the brain develops activity during numerical representations, either in case of non-sumbolic (dots) or symbolic numbers (digits). Cohen et al claimed that various changes and effects occur in the brain, like magnitude changes, format changes, as fMRI adaptations show [8].

Information and Communication Technologies (ICT) have introduced new data related to communication, information, services, entrepreneurship and generally to the improvement of life quality. Consequently, Education has been adapted to the current needs, utilizing ICT tools [9]. The incorporation of ICT tools contributes to the effort to provide equal and qualitative education to all social groups.

In this study we are going to investigate the neuro-cognitive aspects of Developmental Dyscalculia, the attention deficits presented on Dyscalculic children, the components of Working Memory which are related to arithmetic skills and mathematical performance, as well as the possible impact of poor performance in these specific components. 


\section{Neurocognitive Aspects of children with Developmental Dyscalculia.}

Ansari et al introduced an innovative term in the bibliography, neuroeducation, in order to study the field of education and neuroscience [10]. Throughout this review, authors draw attention to how brain mechanisms could affect learning and point out the relation between brain training and cognitive funtions.

Neuroimaging studies support the hypothesis that students with DD present deficits associated to arithmetic learning or mathematical thinking, such as learning math symbols and retrieving arithmetic facts [11]. Deficits in multiple brain regions (mostly in the intraparietal sulcus IPS) as well as deficits in neural plasticity could affect various cognitive functions such as arithmetic skills [12]. Mussolin et al observed differences in brain activation related to symbolic numerical and color distance between DD and control children [13]. Specifically their study revealed lack of left IPS sensivity for dyscalculic children in tasks varying number magnitude.

Diffusion Tensor Imaging (DTI) showed significant differences in white matter integrity between children of typical development and those diagnosed with DD, as well as in Fractional Anisotropy which is related to reduced cognitive performance [14].

According to Huber et al, adults with DD face difficulties in learning new number line layouts or mapping numbers onto space and thus there comes lack in performance in the number line estimation task [15]. For children with DD it could be easier to put analogic numerosities such as spoken numbers in a mental number line, rather than do the same process with symbolic Arabic numbers [16].

Neuroscience findings could positively affect classroom interventions for children with DD [17]. Teachers could take advantage advantage from the finger use for symbolic number representations, as finger-based calculations could be considered as a backup strategy for number processing.

\section{Working Memory Deficits}

Working memory is a mental ability which allow us to control, regulate and keep vigorously relevant information with the aim of doing complicated cognitive tasks such as mathematical processing [18]. Working memory consists of a central executive that is responsible for the whole system, controls its activities, deals with cognitive tasks such as mental arithmetic and problem solving and two slave systems. These are the visual sketchpad and phonological loop which have an active role in verbal and visual/spatial information, respectively [19].

The development of Mathematical skills is based on the capacity and the efficiency of working memory and the executive functions: inhibition, shifting and updating. A meta-analysis study in children between 4 and 12 years confirmed that every component of the working memory system impacts separately on mathematic skills [20]. The working memory component that is mostly related with mathematical performance is verbal updating especially in children with DSM disorders or physical disabilities. After verbal updating ensued the visuospatial sketchpad which was equal to 
the visuospatial updating, the phonological loop and the inhibition which was equal to shifting.

Findings from a study in 12 age children have showed us that spatial working memory processes are closely related with the neural basis of Developmental Dyscalculia, more specifically with the formation of spatial number representations, the storage and retrieval of mathematical information. Also, according to the study children with DD had differences with the activation of the right IPS, the right inferior frontal and the right insula in comparison with the normally achieving controls. The poor central role of the right IPS is associated with the Dysfunctional neural network of spatial wm contributes to DD working memory capacity, spatial number presentation and arithmetic concepts. However, the role of the IPS in these processes is still controversial [21].

Working memory is involved even in very simple mathematical skills and numerical activities such as transcoding numbers. Camo's study in 7-year old children evaluate the working memory capacity on number transcoding from verbal form DD is related to visual spatial memory and inhibition impairment. Low working memory capacity impedes both efficiency and learning of number transcoding to Arabic form. Therefore, difficulties in mathematics may appear from the first acquired knowledge in the early school age of the child. Also, teaching methods should be automatized and retrieval, because working memory is less affected from them, instead of algorithmic [22].

Some studies have demonstrated that low mathematical performance both in adults and children can be occurred when working memory capacity is overloaded, for instance with mental calculations rather than written calculations. Moreover, tasks which require utilizing the central executive component, the phonological loop or the visual-spatial sketchpad simultaneously have negatives effects on the execution of calculation processes.

D'Amico and Guarnera (2005) investigated how the working memory functions affect children with low arithmetical achievement and normal reading abilities compared to normal controls. A group of 9 years old children participated in a study with a series of working memory tasks. Particularly, the study used the central executive functions as the material was both linguistic and numerical, the phonological loop using words, pseudo-words and digits as well as the visual sketchpad using visualspatial sequences and patterns. The results indicated that poor arithmetical abilities turned up in all visual sketchpad tasks and in all the central executive tasks with the exception of phonological loop tasks. However, the only phonological loop measure with low performance was the digit span forward which is explained from the difficulty in recalling numerical information [23].

The verbal short-term storage capacities play a significant role in mathematical disabilities. The study of Attout and Majerus (2015) focus on working memory for serial order information and made a distinction between item and serial order retention capacities in order to gain a deeper understanding of verbal WM impairment in DD. Thirty-two 8 to 12-year-old children participated in the study, the half of them with poor mathematical abilities and the rest of them with the normal mathematical performance, matched on age, IQ, and reading abilities. It was concluded that children 
with DD showed a deficit in a WM task for order information but not in a WM task for item information. Furthermore, only the order WM is related with calculation abilities and still after controlling for item WM capacity [24].

According to Butterworth (2008), there is no correlation between the working memory measures and measures of arithmetic ability. Even though difficulties in working memory come with math difficulties, the fact that forms of working memory provoke the features of dyscalculia does not exist too [25].

\section{$4 \quad$ Attention Deficits}

Some studies present DD as a specific disorder related to basic numerical magnitude and others as a general biological abnormality. These views converge to the heterogeneity in DD's symptoms. In a study with four 8-9 year old children and a set of tasks in order to underline the heterogeneity of DD origins, two cases were connected to variable deficits in the numerical system while the third one suffered from a combination of deficits and the last one was diagnosed with general cognitive impairments only [26].

Learning ability pass through diverse stages. According to Anobile et al (2013), attention system handle the receiving information, maintain or reject it, work out and eventually memorize it. In this investigation, sixty-eight school age typically developing children participated and the results emphasize the connection of attention deficits and math skills [27].

Therefore, two basic axons (executive and attention functions) have been analyzed in a study with 314 Italian students from public primary schools, using two guide tools: Calculation Ability MT Group 6-11 \& Attention and Concentration Battery. Research findings showed that there is an assessing connection between scores in mathematics test and attention skills. A rapid upgrade is observed between attentive rate and calculation ability, at the beginning of the learning procedure. The response time in arithmetic tasks depends on several factors, which are the capacity for awareness, sustained attention and alerting. Math difficulties can be prevented with constant training of the attentive system [28].

In addition, another study focused on the demonstration of attention deficits in DD [29]. Three categories have been mentioned: alerting, orienting and executive procedures. Regions of the right hemisphere (especially in the parietal lobe) are responsible for alerting network control. The alert system activates and maintains the levels of awakeness in the human body. The orienting ability is an important skill in everyday life. Spatial perception is connected to defining horizontal and vertical direction. The executive system is located in the midline frontal areas and includes a cluster of cognitive processes such as organization, discipline, self-regulation, time management, attention and control of impulses. A total of fourteen participants with DD were instructed to put through ANT \& ANT-I tasks. Finally, study results revealed that attention deficits were not related to numerical treatment and were independent from them, as is the case in ADHD. 
Two 10-years old student diagnosed with DD and eight other normallyl developing children participated in Moeller's et al (2009) research, using the Attention test Battery which tested alertness, flexibility and maintained attention. The result of this study revealed that there is no significant correlation between attention deficits and DD [30].

However, the study of Rajaie et al (2011) generally associates the deficiencies in learning mathematics and teaching attention. Fifteen students in the primary school of Tehran participated in the IRAN KIMAT recognition exam and it is eventually clarified that teaching attention plays a pivotal role in the mathematical process [31].

Several studies support that attention depends on the separation of simultaneous processes. Four participants were examined in single-task and dual-task conditions [32]. In the first case, the data representation was depicted linearly, but in the second, as long as attentional resources were increased, the representation of results was displayed in a logarithmic way. Thus, the authors conclude that the attention system mechanism is related to arithmetic magnitude.

The Lindsay's et al (2001) hypothesis claimed that attention deficits are deeply connected to dyscalculia. Researchers examined the relation between attention problems and low performance in mathematics using CPT (Conners Computerized Continuous Performance Test) in a group of 27 Caucasian students with DD. Finally, the results confirmed the initial hypothesis, as there was strong correlation between arithmetic ability and attention difficulties [33].

\section{Assessment of Neurocognitive Impairments via ICT tools.}

In a study with 1,004 primary school children [34], computerized experimental tasks were used in order to investigate the correlation of Developmental Dyscalculia with visuospatial memory and inhibition impairment. Computerized tasks administered were: Simple reaction time, sustained attention, stop-signal task, Animal stroop, Numerical magnitude comparison stroop task, Physical size comparison stroop task, Subitizing, Symbolic magnitude comparison and non-Symbolic magnitude comparison. Justifying current cognitive neuroscience theories, results showed visuospatial short term memory/working memory impairments in dyscalculic children, in contrast to verbal STM/WM where there were no significant differences between DD and control group.

Cangoz et al (2013) developed a computer based screening DD test for 6-9 year old children compatible with android tablets. Technology tools have invaded every aspect of our life at a high level, so tablets have become very popular especially to the children. Furthermore, they are easy to be used by them in the classroom. Based on the neuropsycological correlates of DD, researchers proposed five cognitive tasks which are: dot counting, number comparison, perceptual quantity estimation, number line estimation, and simple arithmetic. The proposed tool consists of a task trial section and a task screening section. In the first, the participants attempt to complete the test as a practice through examples without recording the accuracy of their answers. In the 
other section, the screening test, which is the real test, participants complete the whole tasks in a half an hour. Responses are stored in the tablet for constant processing [35].

A study with 109 participants was conducted in order to investigate the cognitive profile of children with DD and/or ADHD [36]. In order to assess number processing and calculation skills, researchers administered on a computer ZAKERI-R, a neuropsychological battery. The tasks assessing numerical skills were: Dot enumeration, Number comparison, Mixed comparison, Transcoding, Number sets, Number line \& Estimation. Another computer based battery, KITAP, was administered in order to measure alertness, sustained attention and flexibility. The results revealed that while children with DD showed impairments in arithmetic skills, calculations, attention and working memory, those with ADHD had poor performance on attention, alertness and response time tasks.

McCaskey, Von Aster, Tuura \& Kucian (2017) examined magnitude deficits on adolescents with DD using neuro-imaging methods. In order to assess participants' visuo-spatial abilities, they administered three computerized tasks: Visuo-perceptive task, including length estimation and dot comparison tasks, Visuo-cognitive task, where participants are asked to recognize a given figure, hidden among other figures and the Visuo-constructive task, which requires drawing or assembling geometrical figures (including three dimensional). The results showed that the DD group performed significantly worse (in accuracy and reaction time) in complex visuo-spatial tasks, as well as in numerical tasks. However Developmental Dyscalculia and Typically Developing performed similarly on discrete and continuous magnitude tasks, indicating that multiple neuro-cognitive components are related to DD [37].

Based both on the cognitive psychology and neuroscience Karagiannakis and Baccaglini-Frank (2014) designed a tool for identifying the mathematical learning profiles of 10-12 year old children, called the Detecting Difficulties in Mathematics (DeDiMa) battery. This computer-based assessment tool tests four areas through 13 tasks without time limit. Tasks of subitizing-enumeration, number and dots magnitude evaluate core number, tasks of addition and multiplication fact retrieval evaluate memory, tasks of ordinality evaluate visual spatial. Finally, tasks of number lines 01000 , math terms, principal and mental calculations, equations and word problems evaluate reasoning. The tool is accompanied with supplementary cognitive tests which are a visual matrix span tasks, written calculations tasks and a reading fluency standardized test. The aim of this tool, which is in line with the DSM-V, is to outline the student's mathematical profile as well as his performance .Therefore, it can be developed more effective interventions to eliminate the difficulties, through new teaching practices in the class according the individual needs [38].

Rykhlevskaia et al (2009) used neuroimaging analysis techniques in order to find neuroanatomical correlates of DD. Voxel-Based Morphometry analysis (VBM) was used to assess structural impairments in 7 to 9 year old children with DD $(\mathrm{N}=23)$, compared to typically developing children $(\mathrm{N}=24)$. Results showed reduced Gray Matter as well as White Matter volume in posterior brain regions. Furthermore, according to the results, difficulties in numerical operations could be correlated with micro-structural impairments in the right hemisphere of children with DD [39]. 
Rotzer et al (2007) used Voxel Based Morphometry analysis in order to identify brain structure differences, comparing the volume of Gray and White Matter between typically developing children and children with DD. Data used for VBM analysis were collected through magnetic resonance images (MRI). According to the results, children with DD (but no other learning disorders) presented deficits in gray matter volume in the right IPS compared to typically developing children, in contrast with white matter volume, where there was no significant volume differences [40].

In 2009, Kauffman et al used functional Magnetic Resonance Imaging to investigate neural correlates of nosymbolic number magnitude processing in children with DD $(\mathrm{N}=9)$ and controls $(\mathrm{N}=9)$. In contrast with other neuroimaging studies, the results of this study revealed stronger activations in left intraparietal regions in children with DD compared to typically developing [41].

Table 1. Computerized neuropsychological assessment material and neuro-imaging techniques used to identify the cognitive profile of children with Specific Learning Disorder in Mathematics.

\begin{tabular}{|c|c|c|c|c|}
\hline Authors & Year & $\mathbf{N}$ & Assessment Material & Cognitive Measures \\
\hline Askenazi \& Henik & 2010 & 28 & Attention Network Task $(A N T-I)$ & Alertness, Attention \\
\hline Commodari \& Di Blasi & 2014 & 314 & $\begin{array}{l}\text { Attention and Concentration } \\
\text { Battery }\end{array}$ & Attention \\
\hline Guarnera \& D'Amico & 2014 & - & Attenzione e Concentranzione & $\begin{array}{l}\text { Attention, Working } \\
\text { Memory }\end{array}$ \\
\hline $\begin{array}{l}\text { Karagiannakis \& Bac- } \\
\text { caglini-Frank }\end{array}$ & 2014 & 2 & DeDiMa Computerized Battery & $\begin{array}{l}\text { Mathematical Learning } \\
\text { Profiles }\end{array}$ \\
\hline Kaufmann et al & 2009 & 18 & $\mathrm{fMRI}^{1}$ & $\begin{array}{l}\text { Neural Correlates of } \\
\text { non-symbolic number } \\
\text { magnitude processing }\end{array}$ \\
\hline $\begin{array}{l}\text { Kuhn, Ise, Raddatz, } \\
\text { Schwenk \& Dobel }\end{array}$ & 2016 & 109 & $\begin{array}{l}\text { ZAKERI-R Computerized Neuro- } \\
\text { psychological Battery }\end{array}$ & $\begin{array}{l}\text { Attention and Working } \\
\text { Memory }\end{array}$ \\
\hline Kusian et al & 2013 & 47 & Diffusion Tensor Imaging (DTI) & White Matter Volume \\
\hline $\begin{array}{l}\text { McCaskey, Von Aster, } \\
\text { Tuura \& Kucian }\end{array}$ & 2017 & 30 & fMRI & Magnitude Deficits \\
\hline $\begin{array}{l}\text { Moeller, Neuburger, Kauf- } \\
\text { mann, Landerl \& Nuerk }\end{array}$ & 2009 & 10 & Attention Battery & $\begin{array}{l}\text { Alertness, Flexibility } \\
\text { and Maintained Atten- } \\
\text { tion }\end{array}$ \\
\hline Mussolin et al & 2009 & 30 & fMRI & $\begin{array}{l}\text { Reaction Time and } \\
\text { Brain Activation }\end{array}$ \\
\hline Rotzer et al & 2008 & 24 & Voxel Based Morphometry & $\begin{array}{l}\text { Gray and White Matter } \\
\text { Volume }\end{array}$ \\
\hline $\begin{array}{l}\text { Rykhlevskaia, Uddin, } \\
\text { Kondos \& Menon }\end{array}$ & 2009 & 47 & Voxel Based Morphometry & $\begin{array}{l}\text { Gray and White Matter } \\
\text { Volume }\end{array}$ \\
\hline $\begin{array}{l}\text { Szucs, Devine, Soltesz, } \\
\text { Nobes \& Gabriel }\end{array}$ & 2013 & 1004 & Computerized Memory Tasks & Short Term Memory \\
\hline $\begin{array}{l}\text { Träff, Olsson, Östergren \& } \\
\text { Skagerlund }\end{array}$ & 2017 & 4 & Panamath & $\begin{array}{l}\text { Non-Symbolic Number } \\
\text { Comparison }\end{array}$ \\
\hline
\end{tabular}

\footnotetext{
${ }^{1}$ Functional Magnetic Resonance Imaging
} 


\section{Conclusion}

A factor that significantly affects mathematical skills is the neuro-psychological basis of the mathematical knowledge. Specific brain areas are responsible for counting, identification of arithmetic symbols and numerical operations, following algorithms for logical analysis and arithmetic skills in general. Dysfunctions of the left hemisphere may harass the ability to execute arithmetic operations, cause difficulties in maintaining and recalling number facts as well as in understanding number concepts. Neural imaging studies indicate that intraparietal sulcus (IPS) as one of the most critical brain areas for number processing and number comparison.

The relationship between working memory, mathematic ability and the cognitive impairment in children with specific learning disorder in mathematics is intimately related. The central executive and the visual sketchpad have a great importance in arithmetic abilities. Deficient spatial working memory may inhibit the formation of mental number line ,the storage of information and the arithmetic fact retrieval. Therefore, we highlight that the demand on working memory is especially high in acquisition of numerical activities even in the simplest abilities such as transcoding numbers. Poor mathematical performance from a neuropsychological perspective provides knowledge in the cause of the disorder and the way of its enhancement cannot be separated from this perspective.

On dyscalculic children, disorders in attention mechanisms have been observed and simultaneously mathematical skills are severely impaired. By targeting the student population with DD, problems are encountered in serial memory, memorization and executing algorithms. The key to understand how human brain responds to stimulus is to approach via alternative aspects. We are wondering all these factors could have an influence on learning ability. Thus, we pass through different stages and focus on capacities in cognitive functions, working memory and attention deficits, compared to arithmetic skills. The field of attention is a particularly neglected sector, but equally important, due to connectivity between attentional disorders and performace in numerical processes.

The diagnosis should be based on a multidimensional assessment that will detect different mathematical procedures and relevant cognitive skills. mation and Communication Technologies in education provide information for the diagnosis of developmental dyscalculia. Indeed, during the last decade various computerized screening tools have been designed for this purpose.

\section{$7 \quad$ References}

[1] Grotheer, M., Herrmann, K. H., \& Kovács, G. (2016). Neuroimaging evidence of a bilateral representation for visually presented numbers. Journal of Neuroscience, 36(1), 88-97. https://doi.org/10.1523/JNEUROSCI.2129-15.2016

[2] De Smedt, B., Noël, M. P., Gilmore, C., \& Ansari, D. (2013). How do symbolic and nonsymbolic numerical magnitude processing skills relate to individual differences in children's mathematical skills? A review of evidence from brain and behavior. Trends in Neuroscience and Education, 2(2), 48-55. https://doi.org/10.1016/j.tine.2013.06.001 
Paper-Enhanced Assessment Technology and Neurocognitive Aspects of Specific Learning Disorder ..

[3] Kosc, L. (1974). Developmental dyscalculia. Journal of learning disabilities, 7(3), 164177. https://doi.org/10.1177/002221947400700309

[4] American Psychiatric Association. (2013). Diagnostic and statistical manual of mental disorders (5th ed.). Arlington, VA: American Psychiatric Publishing. https://doi.org/10.1176/ appi.books.9780890425596

[5] Devine, A., Soltész, F., Nobes, A., Goswami, U., \& Szücs, D. (2013). Gender differences in developmental dyscalculia depend on diagnostic criteria. Learning and Instruction, 27, 31-39. https://doi.org/10.1016/j.learninstruc.2013.02.004

[6] Rapin, I. (2016). Dyscalculia and the Calculating Brain. Pediatric Neurology, 61, 11-20. https://doi.org/10.1016/j.pediatrneurol.2016.02.007

[7] Bressler, S. L., \& Menon, V. (2010). Large-scale brain networks in cognition: emerging methods and principles. Trends in cognitive sciences, 14(6), 277-290. https://doi.org/10.1016/j.tics.2010.04.004

[8] Cohen Kadosh, R., Bahrami, B., Walsh, V., Butterworth, B., Popescu, T., \& Price, C. J. (2011). Specialization in the human brain: the case of numbers. Frontiers in Human Neuroscience, 5, 62. https://doi.org/10.3389/fnhum.2011.00062

[9] Drigas, A. S., Pappas, M. A., \& Lytras, M. (2016). Emerging Technologies for ICT based Education for Dyscalculia: Implications for Computer Engineering Education. INTERNATIONAL JOURNAL OF ENGINEERING EDUCATION, 32(4), 1604-1610.

[10] Ansari, D., De Smedt, B., \& Grabner, R. H. (2012). Neuroeducation-a critical overview of an emerging field. Neuroethics, 5(2), 105-117. https://doi.org/10.1007/s12152-011-9119-3

[11] Pappas, M. A., \& Drigas, A. S. (2015). ICT Based Screening Tools and Etiology of Dyscalculia. International Journal of Engineering Pedagogy, 5(3).

[12] Iuculano, T. (2016). Neurocognitive accounts of developmental dyscalculia and its remediation. Progress in Brain Research, 227, 305-333. https://doi.org/10.1016/bs.pbr.2016. 04.024

[13] Mussolin, C., De Volder, A., Grandin, C., Schlögel, X., Nassogne, M. C., \& Noël, M. P. (2010). Neural correlates of symbolic number comparison in developmental dyscalculia. Journal of cognitive neuroscience, 22(5), 860-874. https://doi.org/10.1162/jocn. 2009.21237

[14] Kucian, K., Ashkenazi, S. S., Hänggi, J., Rotzer, S., Jäncke, L., Martin, E., \& von Aster, M. (2014). Developmental dyscalculia: a dysconnection syndrome?. Brain Structure and Function, 219(5), 1721-1733.

[15] Huber, S., Sury, D., Moeller, K., Rubinsten, O., \& Nuerk, H. C. (2015). A general numberto-space mapping deficit in developmental dyscalculia. Research in developmental disabilities, 43, 32-42. https://doi.org/10.1016/j.ridd.2015.06.003

[16] Lafay, A., St-Pierre, M. C., \& Macoir, J. (2016). The Mental Number Line in Dyscalculia Impaired Number Sense or Access From Symbolic Numbers?. Journal of learning disabilities, 0022219416640783

[17] Kaufmann, L. (2008). Dyscalculia: neuroscience and education. Educational Research, 50(2), 163-175. https://doi.org/10.1080/00131880802082658

[18] Raghubar, K. P., Barnes, M. A., \& Hecht, S. A. (2010). Working memory and mathematics: A review of developmental, individual difference, and cognitive approaches. Learning and individual differences, 20(2), 110-122. https://doi.org/10.1016/j.lindif.2009.10.005

[19] Rosselli, M., Matute, E., Pinto, N., \& Ardila, A. (2006). Memory abilities in children with subtypes of dyscalculia. Developmental neuropsychology,30(3), 801-818. https://doi.org/10.1207/s15326942dn3003 3

[20] Friso-van den Bos, I., van der Ven, S. H., Kroesbergen, E. H., \& van Luit, J. E. (2013). Working memory and mathematics in primary school children: A meta- 
Paper-Enhanced Assessment Technology and Neurocognitive Aspects of Specific Learning Disorder ...

analysis. Educational research review, 10, 29-44. https://doi.org/10.1016/j.edurev. 2013.05.003

[21] Rotzer, S., Loenneker, T., Kucian, K., Martin, E., Klaver, P., \& Von Aster, M. (2009). Dysfunctional neural network of spatial working memory contributes to developmental dyscalculia. Neuropsychologia, 47(13), 2859-2865. https://doi.org/10.1016/j.neuropsycho $\underline{\operatorname{logia} .2009 .06 .009}$

[22] Camos, V. (2008). Low working memory capacity impedes both efficiency and learning of number transcoding in children. Journal of experimental child psychology, 99(1), 37-57. https://doi.org/10.1016/j.jecp.2007.06.006

[23] D'Amico, A., \& Guarnera, M. (2005). Exploring working memory in children with low arithmetical achievement. Learning and Individual Differences, 15(3), 189-202. https://doi.org/10.1016/j.lindif.2005.01.002

[24] Attout, L., \& Majerus, S. (2015). Working memory deficits in developmental dyscalculia: The importance of serial order. Child Neuropsychology, 21(4), 432-450. https://doi.org/10.1080/09297049.2014.922170

[25] Butterworth, B. (2008). Developmental dyscalculia. Child neuropsychology: Concepts, theory, and practice, 357-374.

[26] Kalbfleisch, L., Lundy, T. J., De Visscher, A., \& Skagerlund, K. (2016). Heterogeneity of developmental dyscalculia: Cases with different deficit profiles.

[27] Anobile, G., Stievano, P., \& Burr, D. C. (2013). Visual sustained attention and numerosity sensitivity correlate with math achievement in children. Journal of experimental child psychology, 116(2), 380-391. https://doi.org/10.1016/j.jecp.2013.06.006

[28] Commodari, E., \& Di Blasi, M. (2014). The role of the different components of attention on calculation skill. Learning and individual differences, 32, 225-232. https://doi.org/10.1016/j.lindif.2014.03.005

[29] Askenazi, S., \& Henik, A. (2010). Attentional networks in developmental dyscalculia. Behavioral and brain functions, 6(1), 2. https://doi.org/10.1186/1744-9081-6-2

[30] Moeller, K., Neuburger, S., Kaufmann, L., Landerl, K., \& Nuerk, H. C. (2009). Basic number processing deficits in developmental dyscalculia: Evidence from eye tracking. Cognitive development, 24(4), 371-386. https://doi.org/10.1016/j.cogdev.2009.09.007

[31] Rajaie, H., Allahvirdiyani, K., Khalili, A., \& Sadeghi, A. (2011). Effect of teaching attention to the mathematic performance of the students with Dyscalculia in the third and fourth grade of elementary school. Procedia-Social and Behavioral Sciences, 15, 3024-3026. https://doi.org/10.1016/j.sbspro.2011.04.236

[32] Anobile, G., Cicchini, G. M., \& Burr, D. C. (2012). Linear mapping of numbers onto space requires attention. Cognition, 122(3), 454-459. https://doi.org/10.1016/j.cognition.2011. 11.006

[33] Lindsay, R. L., Tomazic, T., Levine, M. D., \& Accardo, P. J. (2001). Attentional function as measured by a continuous performance task in children with dyscalculia. Journal of Developmental \& Behavioral Pediatrics, 22(5), 287-292. https://doi.org/10.1097/00004703200110000-00002

[34] Szucs, D., Devine, A., Soltesz, F., Nobes, A., \& Gabriel, F. (2013). Developmental dyscalculia is related to visuo-spatial memory and inhibition impairment. Cortex, 49(10), 26742688. https://doi.org/10.1016/j.cortex.2013.06.007

[35] CANGÖZ, B., Altun, A., Olkun, S., \& Kacar, F. (2013). Computer Based Screening Dyscalculia: Cognitive and Neuropsychological Correlates. TOJET: The Turkish Online Journal of Educational Technology, 12(3).

[36] Kuhn, J. T., Ise, E., Raddatz, J., Schwenk, C., \& Dobel, C. (2016). Basic numerical processing, calculation, and working memory in children with dyscalculia and/or ADHD 
Paper-Enhanced Assessment Technology and Neurocognitive Aspects of Specific Learning Disorder ...

symptoms. Zeitschrift für Kinder-und Jugendpsychiatrie und Psychotherapie. https://doi.org/10.1024/1422-4917/a000450

[37] McCaskey, U., Von Aster, M., Tuura, R. O. G., \& Kucian, K. (2017). Adolescents with Developmental Dyscalculia Do Not Have a Generalized Magnitude Deficit-Processing of Discrete and Continuous Magnitudes. Frontiers in Human Neuroscience, 11. https://doi.org/10.3389/fnhum.2017.00102

[38] Karagiannakis, G., \& Baccaglini-Frank, A. (2014). The DeDiMa battery: a tool for identifying students' mathematical learning profiles. Health Psychology Review, 2(4).

[39] Rykhlevskaia, E., Uddin, L. Q., Kondos, L., \& Menon, V. (2009). Neuroanatomical correlates of developmental dyscalculia: combined evidence from morphometry and tractography. The developing human brain, 172 .

[40] Rotzer, S., Kucian, K., Martin, E., von Aster, M., Klaver, P., \& Loenneker, T. (2008). Optimized voxel-based morphometry in children with developmental dyscalculia. Neuroimage, 39(1), 417-422. https://doi.org/10.1016/j.neuroimage.2007.08.045

[41] Kaufmann, L., Vogel, S. E., Starke, M., Kremser, C., Schocke, M., \& Wood, G. (2009). Developmental dyscalculia: compensatory mechanisms in left intraparietal regions in response to nonsymbolic magnitudes. Behavioral and Brain Functions, 5(1), 35. https://doi.org/10.1186/1744-9081-5-35

\section{Authors}

Marios A. Pappas, Eugenia Malli, Vasiliki Kalpidi, and Athanasios S. Drigas are with the National Center for Scientific Research "Demokritos", Agia Paraskevi, Attica, Greece.

Submitted 30 June 2017. Resubmitted 06 September 2017. Final acceptance 05 February 2018. Final version published as submitted by the authors. 